\title{
Impact of CoreValve size selection based on multi-slice computed tomography on paravalvular leak after transcatheter aortic valve implantation
}

Piotr Chodór ${ }^{1}$, Krzysztof Wilczek², Roman Przybylski ${ }^{3}$, Jan Głowacki, Tomasz Kukulski ${ }^{1}$, Witold Streb ${ }^{1}$, Tomasz Niklewski ${ }^{3}$, Grzegorz Honisz ${ }^{1}$, Przemysław Trzeciak ${ }^{2}$, Tomasz Podolecki ${ }^{1}$, Łukasz Włoch ${ }^{5}$, Marian Zembala ${ }^{3}$, Zbigniew Kalarus ${ }^{1}$

${ }^{1}$ Department of Cardiology, Silesian Center for Heart Diseases, Congenital Heart Diseases and Electrotherapy, Medical University of Silesia, Zabrze, Poland ${ }^{2} 3^{\text {rd }}$ Department of Cardiology, Silesian Center for Heart Diseases,

Medical University of Silesia, Zabrze, Poland

${ }^{3}$ Department of Cardiac Surgery and Transplantology, Silesian Center for Heart Diseases, Medical University of Silesia, Zabrze, Poland

${ }^{4}$ Diagnostic Department, Silesian Center for Heart Diseases, Department of Radiology,

Medical University of Silesia, Poland

${ }^{5}$ Students Scientific Society, Medical University of Silesia, Katowice, Poland

\begin{abstract}
Background: Paravalvular leak (PVL) has significant impact on long-term outcomes in patients after transcatheter aortic valve implantation (TAVI). This study sought to determine whether multi-slice computed tomography (MSCT)-guided valve selection reduces PVL after CoreValve implantation.

Methods: The analysis encompassed 69 patients implanted with CoreValve and were divided into two groups. In Group I (30patients), valve selection was based on standard procedures, in Group II (39patients), on MSCT measurements. Paravalvular leak was assessed with angiography and echocardiography.

Results: Multi-slice computed tomography results influenced a change of decision as to the size of the implanted valve in 12 (30.9\%) patients in Group II and would have caused the decision to change in 9 (37.5\%) patients in Group I. The degree of oversizing in Group I and II was $12.8 \% \pm$ $\pm 7.6 \%$ vs. $18.6 \% \pm 5.1 \%(p=0.0006)$, respectively. The oversizing among the patients with leak degree of $0-1$ and $\geq 2$ was $18.1 \% \pm 6.0 \%$ and $12.8 \% \pm 7.4 \%(p=0.0036)$. Angiographic assessment indicated post-procedural PVL $\geq 2$ in $50 \%$ of patients in Group I and $20.5 \%$ in Group II ( $p=0.01)$, while echocardiographic assessment indicated the same in $73.3 \%$ of patients in Group I and $45.6 \%$ in Group II ( $p=0.0136)$. The composite endpoint occurred in 26.6\% (8/30) patients in Group I vs. $5.1 \%$ (2/39) patients in Group II ( $p=0.0118)$.

Conclusions: Selecting the CoreValve device based on MSCT resulted in smaller rates of PVL and less frequent composite endpoint. In 1/3 of patients MSCT led to a change of the valve size. The degree of oversizing had a significant impact on PVL. (Cardiol J 2017; 24, 5: 467-476)
\end{abstract}

Key words: paravalvular leak, aortic valve stenosis, transcatheter aortic valve implantation, CoreValve, multi-slice computed tomography

Address for correspondence: Piotr Chodór, MD, PhD, Department of Cardiology, Silesian Center for Heart Diseases, Congenital Heart Diseases and Electrotherapy, Medical University of Silesia, ul. Skłodowskiej-Curie 9, 41-800 Zabrze, Poland, tel: +48 32 2713414, fax: +48 32 3733792, e-mail: chodor_piotr@go2.pl

Received: 06.05.2016 Accepted: 23.11.2016 


\section{Introduction}

Transcatheter aortic valve implantation (TAVI) is a new, fast-developing method of treatment for patients with severe aortic valve stenosis, high surgical risk and for inoperable patients [1]. Valves are generally implanted surgically in patients with lower surgical risk [2]. One of the limitations of TAVI is its association with paravalvular leak (PVL), which in future may hamper the spread of the procedure to patients with low surgical risk.

Paravalvular leak occurs in most patients after TAVI [3, 4]. The actual percentage of PVL varies greatly and depends on assessment methods and criteria, as well as the length of post-TAVI followup. PVL is usually assessed with echocardiography, although this method presents some limitations associated with the eccentric and "spray" nature of PVL [5]. At discharge, PVL is present in 41-94\% of patients (of whom in up to $24 \% \mathrm{PVL}$ is moderate to severe) [5]. In the first randomized TAVI trial, PARTNER, PVL was diagnosed in a total of $88 \%$ of patients, of whom $11.8-12.2 \%$ had moderate to severe PVL $[6,7]$. PVL assessed with angiography immediately after implantation occured in $60.7-94.0 \%$ of patients [8-11]. Even mild PVL may have a negative impact on survival after TAVI $[3,12,13]$.

Paravalvular leak may be caused by valve malpositioning, undersizing, or the presence of calcifications. Valve selection is based on measurements obtained from echocardiography, angiography and multi-slice computed tomography (MSCT). The importance of MSCT (in TAVI patients) in valve selection has been shown by many researchers who demonstrated that MSCT annulus measurements help to predict PVL $[14,15]$. Moreover, appropriate degree of oversizing based on aortic valve area may help to reduce PVL when implanting the balloon-expandable Edwards Sapien (ES) valve. Binder et al. [16] proposed an algorithm in case of ES valve implantation. However, the mechanisms of implantation of the balloon-expandable and the self-expandable systems are different, and necessitate different algorithms.

The aim of the study was to determine whether, compared with the standard procedure, valve selection based on the measurement of diameter calculated from the perimeter of annulus obtained from MSCT test reduces PVL after the implantation of the self-expandable CoreValve device (Medtronic, Mineapolis, MN, USA).

\section{Methods}

Initially, the retrospective analysis encompassed 118 patients who underwent TAVI between November 26, 2008 and February 4, 2014. The first 12 patients were excluded in order to minimize the learning curve effect.

The exclusion criteria were: no effective implantation $(\mathrm{n}=3)$, implantation of a prosthesis other than the self-expanding CoreValve (Accurate: $\mathrm{n}=9$, ES: $n=28)$, valve-in-valve procedure $(n=3)$, valve implantation inconsistent with the schedule $(\mathrm{n}=1)$, lack of or uninterpretable post-procedural aortography $(\mathrm{n}=5)$. The final analysis encompassed 69 patients with severe aortic stenosis, implanted with the self-expandable CoreValve prosthesis.

Until May 2011, the operators based their choice of valve on the available annulus measurements obtained from transthoracic (TTE) and transesophageal (TEE) echocardiography and MSCT (annulus measurement in oblique sagittal view). Since May 2011, the choice of the implanted valve was based on diameter of aortic valve annulus calculated from its perimeter in MSCT test.

All the patients expressed their written informed consent concerning TAVI procedure.

\section{Study endpoints}

The composite endpoint consisted of one or more of the following events: immediate procedural death, annulus rupture, valve migration/embolisation, coronary obstruction and PVL $>2$ assessed with angiography. Other periprocedural and inhospital complications were analyzed: procedural mortality, TAV-in-TAV deployment, periprocedural myocardial infarction, cardiac pacemaker implantation. The definitions of the above-mentioned complications were consistent with the Valve Academic Research Consortium (VARC) 2 definitions.

\section{MSCT-assessment and valve selection}

All MSCTs were performed at the Center and were subsequently assessed at the Medtronic Central Laboratory (CoreLAB) using 3mensio Valves $^{\mathrm{TM}}$ software (3mensio Medical Imaging BV Bilthoven Netherland). Retrospectively, 24 MSCT tests from Group I were available for repeated evaluation.

In all patients, MSCT was performed with a Siemens apparatus (Sensation 64, Siemens Medical, Erlangen, Germany) according to the protocol used at our Center. 
Table 1. Oversizing recommended by Medtronic.

\begin{tabular}{lcccc}
\hline Valve & $\begin{array}{c}\text { Annulus/perimeter } \\
- \text { minimum }\end{array}$ & Oversizing & $\begin{array}{c}\text { Annulus/perimeter } \\
\text { - maximum }\end{array}$ & Oversizing \\
\hline 26 (perimeter $81.6 \mathrm{~mm})$ & $20(62.8 \mathrm{~mm})$ & $29.9 \%$ & $23(72.2 \mathrm{~mm})$ & $13.0 \%$ \\
29 (perimeter $91.1 \mathrm{~mm})$ & $23(72.2 \mathrm{~mm})$ & $26.2 \%$ & $27(84.8 \mathrm{~mm})$ & $7.4 \%$ \\
31 (perimeter $97.3 \mathrm{~mm})$ & $26(81.6 \mathrm{~mm})$ & $19.2 \%$ & $29(91.1 \mathrm{~mm})$ & $6.8 \%$ \\
\hline
\end{tabular}

Next, a $10 \mathrm{~mL}$ bolus of non-ionic, iodine-based, initially of low-osmolar and finally of iso-osmolar contrast medium was injected into the right basilic vein at $4.5-5 \mathrm{~mL} / \mathrm{s}$. After calculating the delay of contrast enhancement in the aortic bulb, contrastenhanced scanning was performed with approx. $140 \mathrm{~mL}$ of contrast medium at $4.5-5 \mathrm{~mL} / \mathrm{s}$. A short section with the aortic valve was scanned with retrospective electrocardiographic gating. Next, with the same volume of contrast agent, the rest of the patient's body was examined from the base of the neck to $1 / 3$ height.

After obtaining the raw data, the valve area was reconstructed in the systolic and diastolic phase $(30 \%, 60 \%$ and $70 \%$ of R-R intervals). Further calculations necessary for aortic valve implantation were performed at the CoreLab according to the protocol. The aortic root measurements were performed in systole $-30 \%$ of R-R interval. The measurements involved the annulus, the bulb, the ascending aorta and the left ventricular outflow tract (LVOT); peripheral arteries were assessed for vascular access.

Until 2011, we received reports on valve parameters: single-plane (sagittal view) annulus measurements, width and height of the aortic bulb, sino-tubular junction size, ascending aorta, LVOT and the peripheral artery diameter. Since 2011, additional data was received regarding the minimum and maximum annulus size and the aortic valve annulus perimeter.

\section{Valve selection}

The patients $(\mathrm{n}=69)$ were divided into two groups. Group I $(n=30)$ was composed of patients in whose case the valve was selected based on annulus diameter assessed by TTE, TEE and MSCT (sagittal view). In Group I, the valve was selected based on the annulus diameter and the Medtronic recommendations (Table 1$)$. Group II $(n=39)$ encompassed patients in whom valve size selection was based on the diameter of aortic valve annulus calculated from its perimeter in MSCT test.

Multi-slice computed tomography-based annulus perimeter served to calculate the annulus

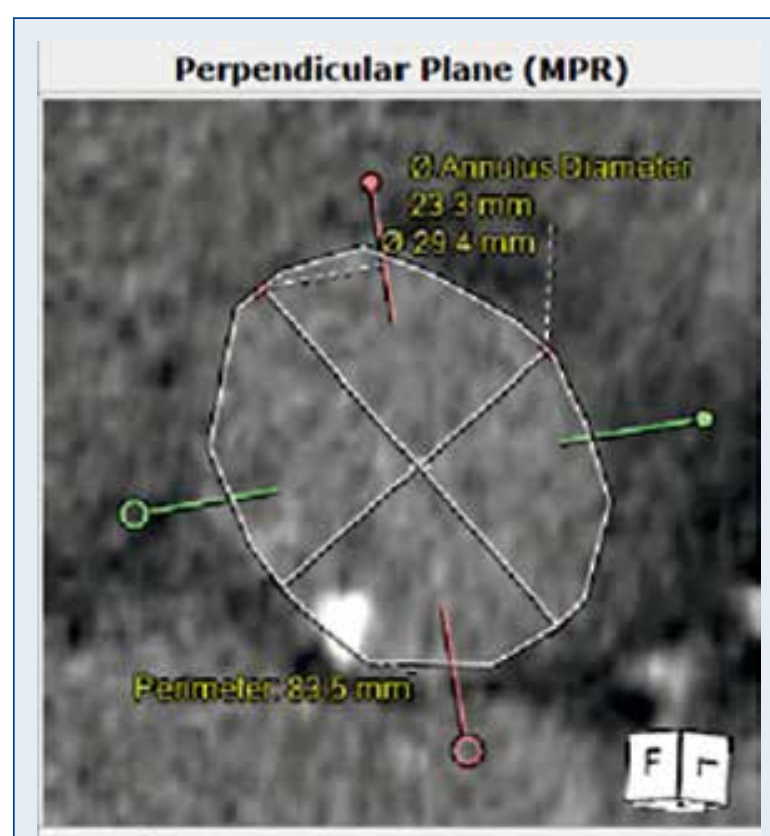

Mean diameter $=26.6 \mathrm{~mm}$

Figure 1. Multi-slice computed tomography-based annulus measurement.

diameter according to the following formula: annulus perimeter/3.14, assuming a round shape of the annulus (Fig. 1).

The annulus diameter was also calculated as an arithmetic mean value of two MSCT-based minimum and maximum annulus diameters. The estimated oversizing was defined as oversizing of the base of the CoreValve device at the level of the native annulus (Fig. 2). Oversizing was expressed according to the arithmetical formula: [ 1 - (perimeter the base of CoreValve / perimeter of the annulus) $] \times 100$ [\%]. Any positive value was considered oversizing, while any negative value was considered undersizing. In reality, optimal valve positioning is $4-8 \mathrm{~mm}$ below the native annulus. In consideration of this and because of the conical shape of the CoreValve base, the degree of its oversizing was smaller than assumed (Fig. 2). 


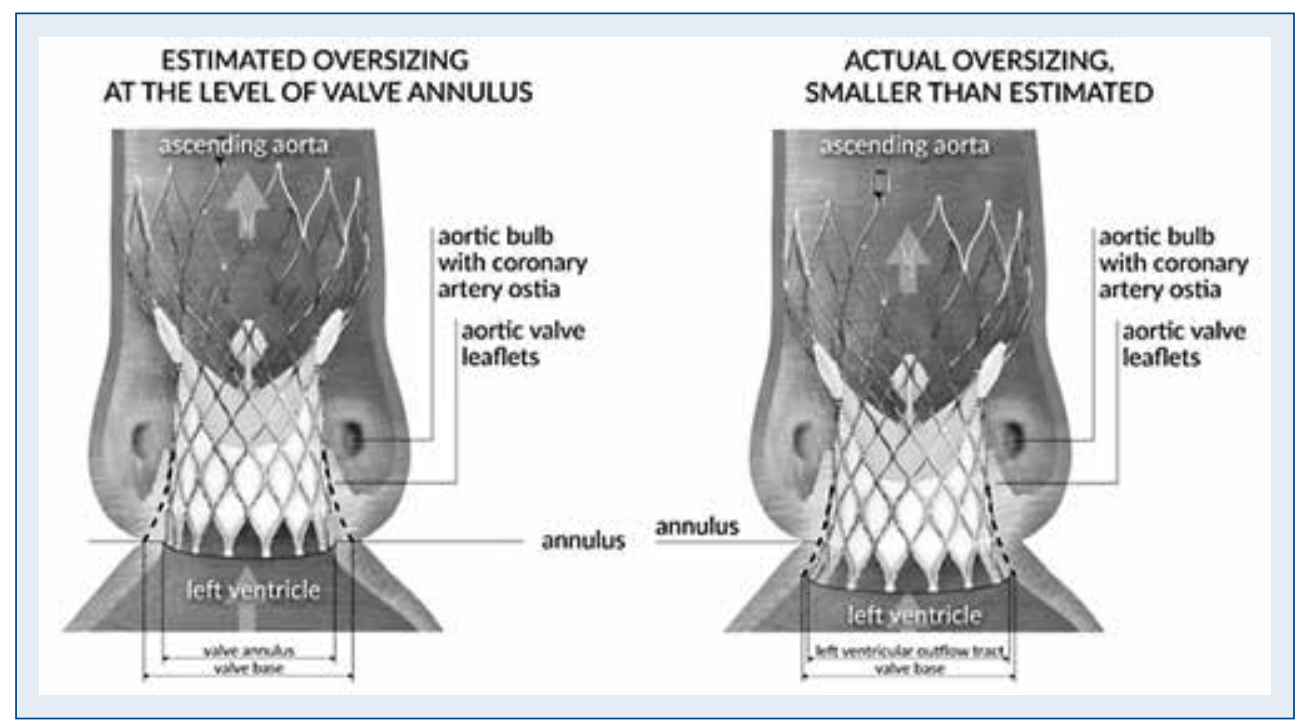

Figure 2. Estimated and actual oversizing of the CoreValve self-expandable device.

In Group I, valve selection was based on TTE/ /TEE and MSCT results, with the aortic annulus diameter being assessed only in sagittal view. TEE was performed in all but 3 patients in Group I during TAVI, prior to valve implantation with a view to assess its anatomy (including the annulus size) final decision as to the implanted valve size was made.

\section{Angiography-based PVL assessment}

The angiography-based PVL assessment was based on aortic valve insufficiency score proposed by Sellers et al. [17]. Aortography was performed after TAVI, in the same or approximately the same view as the one in which the valve was implanted. $20 \mathrm{~mL}$ of contrast medium were injected at $10 \mathrm{~mL} / \mathrm{s} 450 \mathrm{psi}$. Independent assessment was performed by three experienced invasive cardiologists. Divergent opinions concerned $25(36.2 \%)$ patients; and the final decision was jointly made.

\section{Echocardiography-based PVL assessment}

The examination was performed by experienced echocardiographers using the Philips iE 33 xMATRIX and GE Vivid e9 apparatus. The diameter of the aortic valve annulus was defined as the distance between hinge points of the aortic valve semilunar cusps. It was measured along the long axis during the final phase of ventricular systole, as recommended in publications [18, 19]. The degree of PVL was assessed by Doppler echocardiography, according to the VARC I grading criteria: 0 - absent, 1 - trace, 2 - mild, 3 - moderate,
4 - severe. PVL was assessed by echocardiography before discharge.

\section{Statistical analysis}

The obtained data were presented as means and standard deviations (SD). To assess the statistical significance of differences between groups, twotailed Student's t-test for independent variables was used. The Kolmogorov-Smirnov test was used to verify the normality of distribution and Fisher's F-test to assess the equality of variance. The differences between structure indices were assessed with Student's t-test for independent samples.

\section{Results}

There were no statistically significant differences between the two groups with regard to the investigated clinical parameters (Table 2).

Echocardiographic and MSCT data are presented in Table 3.

In Group I, TTE and MSCT data (one plane) did not differ significantly: $23.0 \pm 1.9 \mathrm{~mm}$ vs. $23.4 \pm$ $2.8 \mathrm{~mm}(\mathrm{p}=0.2398)$. The same was true in Group II: $22.9 \pm 2.3 \mathrm{~mm}$ vs. $22.7 \pm 2.4 \mathrm{~mm}(\mathrm{p}=0.7082)$.

Although the mean TTE and MSCT measurements were not significantly different, MSCT results influenced the change of decision as to the size of the implanted valve in 12 (30.9\%) patients in Group II. In 12 patients, echocardiography suggested the choice of a smaller valve, while MSCT (diameter calculated from the perimeter obtained from MSCT) pointed to bigger valves according to the recommendations. 
Table 2. Patient characteristics.

\begin{tabular}{|c|c|c|c|}
\hline & $\begin{array}{l}\text { Group I }(n=30) \\
\text { TTE/TEE/MSCT }\end{array}$ & $\begin{array}{c}\text { Group II }(n=39) \\
\text { MSCT }\end{array}$ & $\mathbf{P}$ \\
\hline Age [years] & $77.4 \pm 7.4$ & $76.7 \pm 9.3$ & 0.7452 \\
\hline Male & $18(60 \%)$ & $24(61.5 \%)$ & 0.8993 \\
\hline Body mass index & $28.2 \pm 3.4$ & $28.3 \pm 5.6$ & 0.9422 \\
\hline Diabetes & $12(40.0 \%)$ & $16(41.0 \%)$ & 0.9332 \\
\hline Arterial hypertension & $22(73.3 \%)$ & $30(76.9 \%)$ & 0.7325 \\
\hline Smoking history & $10(33.3 \%)$ & $17(43.9 \%)$ & 0.3715 \\
\hline Chronic obstructive pulmonary disease & $8(26.7 \%)$ & $11(28.2 \%)$ & 0.8900 \\
\hline NYHA class I & $1(3.3 \%)$ & $0(0.0 \%)$ & 0.2510 \\
\hline NYHA class II & $12(40.0 \%)$ & $12(30.8 \%)$ & 0.4264 \\
\hline NYHA class III & $16(53.4 \%)$ & $23(59.0 \%)$ & 0.6418 \\
\hline NYHA class IV & $1(3.3 \%)$ & $4(10.2 \%)$ & 0.2717 \\
\hline History of cerebrovascular accident or TIA & $4(13.3 \%)$ & $7(17.9 \%)$ & 0.6045 \\
\hline Prior coronary artery bypass graft & $11(36.7 \%)$ & $13(33.3 \%)$ & 0.7688 \\
\hline Valve surgery & $3(10.3 \%)$ & $2(5.1 \%)$ & 0.4122 \\
\hline Porcelain aorta & $4(13.3 \%)$ & $8(20.5 \%)$ & 0.4339 \\
\hline Pulmonary hypertension (> $60 \mathrm{~mm} \mathrm{Hg}$ ) & $2(6.7 \%)$ & $6(15.4 \%)$ & 0.2636 \\
\hline Prior implantation of a cardiac stimulator/ICD/CRT-D & $11(36.6 \%)$ & $8(20.5 \%)$ & 0.1376 \\
\hline Prior myocardial infarction & $14(46.7 \%)$ & $13(33.3 \%)$ & 0.2582 \\
\hline Prior balloon aortic valvuloplasty & $7(23.3 \%)$ & $4(10.3 \%)$ & 0.1437 \\
\hline Prior $\mathrm{PCl}$ within the previous 3 months & $6(20.0 \%)$ & $13(17.9 \%)$ & 0.8249 \\
\hline Non-cardiac vascular lesions & $16(53.3 \%)$ & $13(33.3 \%)$ & 0.0952 \\
\hline Kidney failure [GFR $<60 \mathrm{~mL} / \mathrm{min} / 1.73 \mathrm{~m}^{2}$ ] & $14(48.3 \%)$ & $20(55.6 \%)$ & 0.5472 \\
\hline Atrial fibrillation & $5(16.6 \%)$ & $6(12.8 \%)$ & 0.6563 \\
\hline Standard EuroSCORE & $11.3 \pm 2.9$ & $10.2 \pm 2.2$ & 0.0864 \\
\hline Logistic EuroSCORE [\%] & $26.7 \pm 16.5$ & $20.5 \pm 10.7$ & 0.0638 \\
\hline STS risk score & $6.7 \pm 8.2$ & $5.6 \pm 3.1$ & 0.4302 \\
\hline NT-proBNP [pg/mL] & $3843.7 \pm 5880$ & $3286.5 \pm 6300$ & 0.7118 \\
\hline
\end{tabular}

CRT-D — cardiac resynchronization therapy defibrillator; GFR - glomerular filtration rate; ICD — implantable cardioverter defibrillator; MSCT - multi-slice computed tomography; NT-proBNP - N-terminal natriuretic propeptide type B; NYHA — New York Heart Association; $\mathrm{PCl}$ - percutaneous coronary intervention; STS - Society of Thoracic Surgeons; TIA - transient ischemic attack; TEE - transesophageal echocardiography; TTE — transthoracic echocardiography

According to the recommendations in Group I, if the valve selection had involved the diameter calculated from the perimeter of valve annulus obtained from MSCT test, it would have been necessary to change the decision concerning implantated valves: $6(25 \%)$ patients would have needed bigger valve, $1(4.1 \%)$ patient smaller, and $2(8.2 \%)$ patients would have not qualified for valve implantation because of the annulus size.

Procedure-related data are presented in Table 4.

\section{PVL immediately after the procedure}

Angiographic assessment immediately after the procedure showed that a tight valve with no leak was more frequent in Group II than in Group I: $15(38.5 \%)$ vs. $5(16.7 \%)$ patients respectively
( $\mathrm{p}=0.0479)$. PVL of grade 3 according to Sellers' criteria was less frequent in Group II than in Group I: $2(5.1 \%)$ vs. 6 (20\%); however, the difference was of borderline statistical significance $(\mathrm{p}=0.0552)$. Also PVL grade $\geq 2$ was significantly less frequent in Group II than in Group I (Table 5). Post-procedural echocardiographic assessment also confirmed that PVL occurred significantly less frequently in Group II (Table 5).

The mean degree of oversizing in relation to leak grade $0,1,2,3$ was $19.0 \%, 17.3 \%, 13.9 \%$, and $11.5 \%$, respectively. Significant statistical differences were found in oversizing among patients with the leak of $0^{\text {th }}$ and $3^{\text {rd }}$ degree (respectively 19.0 $\pm 5.3 \%$ vs. $11.5 \pm 6.6 \%, \mathrm{p}=0.0082), 0^{\text {th }}$ and $2^{\text {nd }}$ degree $(19.0 \pm 5.3 \%$ vs. $13.9 \pm 7.7 \%, \mathrm{p}=0.0286)$ 
Table 3. Echocardiographic and MSCT data.

\begin{tabular}{|c|c|c|c|}
\hline & $\begin{array}{l}\text { Group I }(\mathbf{n}=30) \\
\text { TTE/TEE/MSCT }\end{array}$ & $\begin{array}{l}\text { Group II }(n=39) \\
\text { MSCT }\end{array}$ & $\mathbf{P}$ \\
\hline \multicolumn{4}{|l|}{ Echocardiographic data } \\
\hline Ejection fraction [\%] & $42.4 \pm 11.2$ & $47.0 \pm 12.9$ & 0.1210 \\
\hline Mean aortic transvalvular gradient $[\mathrm{mm} \mathrm{Hg}]$ & $50.0 \pm 17.9$ & $48.6 \pm 16.6$ & 0.7629 \\
\hline Annulus size in TTE $[\mathrm{mm}]$ & $23.0 \pm 1.9$ & $22.9 \pm 2.3$ & 0.8377 \\
\hline Annulus size in TEE [mm] & $23.7 \pm 2.3^{1}$ & $22.9 \pm 2.9^{2}$ & 0.3064 \\
\hline Aortic valve area $\left[\mathrm{cm}^{2}\right]$ & $0.60 \pm 0.17$ & $0.64 \pm 0.18$ & 0.3898 \\
\hline Aortic valve area index $\left[\mathrm{cm}^{2} / \mathrm{m}^{2}\right]$ & $0.35 \pm 0.10$ & $0.37 \pm 0.14$ & 0.6005 \\
\hline Pulmonary hypertension (> $60 \mathrm{~mm} \mathrm{Hg}$ ) & $2(6.7 \%)$ & $6(15.4 \%)$ & 0.2636 \\
\hline \multicolumn{4}{|l|}{ MSCT data } \\
\hline Annulus size MSCT (one plane) [mm] & $23.4 \pm 2.8$ & $22.7 \pm 2.4$ & 0.2415 \\
\hline Minimum annulus size $[\mathrm{mm}]$ & $22.8 \pm 2.3^{*}$ & $21.3 \pm 2.3$ & 0.0146 \\
\hline Maximum annulus size $[\mathrm{mm}]$ & $27.7 \pm 3.0^{*}$ & $26.5 \pm 2.3$ & 0.0787 \\
\hline Annulus perimeter $[\mathrm{mm}]$ & $80.7 \pm 6.7^{*}$ & $75.8 \pm 6.8$ & 0.0700 \\
\hline Annulus size calculated with the formula: $\min +\max / 2[\mathrm{~mm}]$ & $25.1 \pm 2.6^{*}$ & $23.8 \pm 2.1$ & 0.0333 \\
\hline Perimeter-derived annulus size (annulus perimeter/3.14) [mm] & $24.8 \pm 5.2^{*}$ & $23.6 \pm 4.1$ & 0.3130 \\
\hline Degree of oversizing [\%] & $12.8 \pm 7.6^{*}$ & $18.6 \pm 5.1$ & 0.0006 \\
\hline $\begin{array}{l}\text { Number of patients in whom valve size was changed } \\
\text { based on MSCT results }\end{array}$ & & $12(35.0 \%)$ & \\
\hline
\end{tabular}

*Data from 24 patients; ${ }^{*} 27$ patients; ${ }^{2} 15$ patients; MSCT — multi-slice computed tomography; TTE — transthoracic echocardiography; TEE — transesophageal echocardiography

Table 4. Procedure-related data.

\begin{tabular}{|c|c|c|c|}
\hline & $\begin{array}{l}\text { Group I }(n=30) \\
\text { TTE/TEE/MSCT }\end{array}$ & $\begin{array}{c}\text { Group II }(n=39) \\
\text { MSCT }\end{array}$ & $\mathbf{P}$ \\
\hline \multicolumn{4}{|l|}{ Anesthesia: } \\
\hline General & $26(86.7 \%)$ & $17(43.6 \%)$ & 0.0431 \\
\hline Local & $4(13.3 \%)$ & $17(43.6 \%)$ & 0.1332 \\
\hline Local with conversion to general & $0(0 \%)$ & $5(12.8 \%)$ & 0.2422 \\
\hline \multicolumn{4}{|l|}{ Access: } \\
\hline Transfemoral & $21(70.0 \%)$ & $28(71.8 \%)$ & 0.8702 \\
\hline Transsubclavian & $8(26.7 \%)$ & $10(25.6 \%)$ & 0.9178 \\
\hline Transaortic & $1(3.3 \%)$ & $1(2.6 \%)$ & 0.8637 \\
\hline Percutaneous & $8(26.7 \%)$ & $18(46.2 \%)$ & 0.0976 \\
\hline Surgical & $22(73.3 \%)$ & $21(53.8 \%)$ & 0.0976 \\
\hline \multicolumn{4}{|l|}{ Valve size: } \\
\hline 26 & $8(26.7 \%)$ & $12(30.8 \%)$ & 0.7030 \\
\hline 29 & $16(53.3 \%)$ & $17(43.6 \%)$ & 0.4239 \\
\hline 31 & $6(20.0 \%)$ & $10(25.6 \%)$ & 0.5847 \\
\hline Balloon postdilatation & $3(10.0 \%)$ & $8(20.5 \%)$ & 0.2375 \\
\hline Implantation depth [mm] — angiographic assessment & $5.9 \pm 3.57$ & $5.8 \pm 3.78$ & 0.9523 \\
\hline Number of proctored procedures & $21(70 \%)$ & $0(0 \%)$ & $<0.001$ \\
\hline
\end{tabular}

MSCT — multi-slice computed tomography; TTE — transthoracic echocardiography; TEE — transesophageal echocardiography 
Table 5. Paravalvular leak in angiographic and echocardiographic assessment.

\begin{tabular}{lccc}
\hline & $\begin{array}{c}\text { Group I }(\mathbf{n}=30) \\
\text { TTE/TEE/MSCT }\end{array}$ & $\begin{array}{c}\text { Group II }(\mathbf{n}=39) \\
\text { MSCT }\end{array}$ \\
\hline PVL in angiographic assessment & & & \\
0 & $5(16.7 \%)$ & $15(38.5 \%)$ & 0.0479 \\
1 & $10(33.3 \%)$ & $16(41.0 \%)$ & 0.5128 \\
2 & $9(30 \%)$ & $6(15.4 \%)$ & 0.1450 \\
3 & $6(20 \%)$ & $2(5.1 \%)$ & 0.0552 \\
4 & $0(0 \%)$ & $0(0 \%)$ & NS \\
$\geq 2$ & $15(50 \%)$ & $8(20.5 \%)$ & 0.0100 \\
PVL assessed with echocardiography & & $9(23.1 \%)$ & 0.7570 \\
Absent & $6(20.0 \%)$ & $13(33.3 \%)$ & 0.0079 \\
Trace & $2(6.7 \%)$ & $22(56.4 \%)$ & 0.0136 \\
Absent/trace & $8(26.7 \%)$ & $13(33.3 \%)$ & 0.1614 \\
Mild & $15(50.0 \%)$ & $4(10.6 \%)$ & 0.2742 \\
Moderate & $6(20.0 \%)$ & $0(0 \%)$ & 0.2532 \\
Severe & $1(3.3 \%)$ & & \\
\hline
\end{tabular}

MSCT — multi-slice computed tomography; TTE — transthoracic echocardiography; TEE — transesophageal echocardiography

and patients with the leak of $0^{\text {th }}-1^{\text {st }}$ and $\geq 2^{\text {nd }}$ degree $(18.1 \pm 6.0 \%$ vs. $12.8 \pm 7.4 \%, \mathrm{p}=0.0036)$. The degree of oversizing differed significantly between Group I and II and was, respectively, $12.8 \pm 7.6 \%$ vs. $18.6 \pm 5.1 \%, p=0.0006$ (Table 3 ).

\section{Clinical course after TAVI}

There were $3(10 \%)$ in-hospital deaths in Group I and $1(2.6 \%)$ in Group II. One of the three deaths in Group I was caused by heart failure attributable to PVL grade 3. Another patient developed septic shock and multiple organ dysfunction syndrome. In that patient, TAVI was performed in a very poor clinical condition following an earlier valvuloplasty. The third patient died due to cardiac tamponade during CRT-D implantation several days after TAVI.

In Group II, 1 patient with a moderate leak died due to post-operative vascular complications. No annulus rupture, valve dislocation, or coronary flow limitation occurred in either group. The composite endpoint occurred in 8 patients in Group I and 2 in Group II ( $p=0.0118)$. The percentages of necessary implantation of cardiac stimulation systems were similar in both groups (Table 6).

\section{Discussion}

The impact of PVL on the outcome has been reported in a number of recent publications. Based on their PARTNER randomized trial, Kodali et al. [3] proved that even a mild PVL following the implantation of a balloon-expandable valve affects 3 -year survival. Schewel et al. [12] also showed that PVL $\geq 2+$ in patients after implantation of self-expandable and balloon-expandable heart valves was associated with a considerable increase in 6-month mortality and a significant increase in $\mathrm{N}$-terminal natriuretic propeptide type B levels. Hayashida et al. [13] concluded that even PVL $=2$ negatively affects the outcome for both CoreValve and ES devices. PVL is attributable to numerous factors, mainly the presence of calcifications, depth of valve implantation, the angle between the LVOT and the aorta, and valve undersizing $[8,11,12,20-23]$.

This study demonstrates that valve selection based on the measurements of diameter and annulus perimeter obtained from MSCT test may increase the percentage of tight CoreValve implantations and reduce the percentage of significant leaks in comparison to the standard procedures. It also presents one of the highest rates of patients with no leak after CoreValve implantation published so far [5]. Valve perimeter was chosen as the base for calculation of the annulus diameter because it had less variability across the cardiac cycle, which gives a more precise reading than area measurements [15]. Also, because of the fact that CoreValve adapts itself to the annulus of the native valve, the calculation of perimeter is more useful here. In the work of Von Aspern et al. [24] the diameter 
Table 6. Clinical course.

\begin{tabular}{lccc}
\hline & $\begin{array}{c}\text { Group I }(\mathbf{n}=30) \\
\text { TTE/TEE/MSCT }\end{array}$ & $\begin{array}{c}\text { Group II (n= } \\
\text { MSCT }\end{array}$ \\
\hline Immediate procedural death & 0 & 0 & NS \\
Procedural mortality & $3(10 \%)$ & $1(2.6 \%)$ & 0.1930 \\
Annular rupture & 0 & 0 & $\mathrm{NS}$ \\
Valve migration/valve embolisation & 0 & 0 & $\mathrm{NS}$ \\
Coronary obstruction & 0 & 0 & $\mathrm{NS}$ \\
TAV-in-TAV deployment & 0 & $\mathrm{NS}$ \\
Perioperative infarction & 0 & 0 & $\mathrm{NS}$ \\
Implantation of a cardiac stimulation system & $9(30 \%)$ & $10(25.6 \%)$ & 0.6850 \\
DDDR & $6(23.0 \%)$ & $9(23.1 \%)$ & 0.9922 \\
VVIR & $2(6.7 \%)$ & $0(0 \%)$ & 0.1009 \\
CRT-D & $1(3.3 \%)$ & $1(2.6 \%)$ & 0.8637 \\
PVL $\geq 2+$ in angiographic assessment & $6(20 \%)$ & $2(5.1 \%)$ & 0.0552 \\
Composite endpoint & $8(26.6 \%)$ & $2(5.1 \%)$ & 0.0118 \\
\hline
\end{tabular}

CRT-D — cardiac resynchronization therapy defibrillator; DDDR — dual chamber rate adaptive pacemaker; MSCT — multi-slice computed tomography; PVL — paravalvular leak; TTE — transthoracic echocardiography; TEE — transesophageal echocardiography; VVIR — single chamber sensor-based, rate-modulating pacemaker

calculated from the surface area of the valve was smaller than the diameter calculated from the perimeter in MSCT test. In case of balloon-expanded valve, the valve adjusts the native annulus to its round shape. In this case it is more appropriate to calculate the diameter of annulus from the surface area of the valve.

The main disadvantage of MSCT valve measurement is the fact that in some patients the quality of the examination is not sufficient for reliable measurements. In the present study, this was the case in $15.2 \%$ of patients. Factors affecting quality of the examination include heart rate, heart rhythm disorders and obesity.

What were the deciding factors for better valve adjustment and smaller PVL in Group II? TTE, TEE and MSCT examinations determined the aortic annulus size in one dimension only and may be adequate only in cases of round-shaped aortic valves. According to Schultz et al. [25] though, the aortic valve is round in $50 \%$ of cases. In patients with elliptic valves, two measurements are available: minimum and maximum. In such situations, decisions based on measuring only one dimension, if it is the smaller dimension, tend to lead to the selection of a smaller valve and the occurrence of PVL.

In this study, echocardiography measurements yielded smaller values than those from MSCT. TTE and MSCT (one plane) results did not differ significantly. Most probably, this ambiguous results were due to the small size of the investigated groups. The less frequent occurrence of PVL was determined by the change in decision regarding valve size selection based on the measurement of annulus diameter based on perimeter obtained from MSCT test in comparison with the measurement of the annulus diameter using TTE. This concerned $30.9 \%$ of patients in Group II, and all cases which involved a change to a larger valve. Retrospective analysis of MSCT images in Group I showed that as many as $37.0 \%(9 / 24)$ should have received a different size of valve. Similar conclusions have been reached by Mylotte et al. [26]. He found that in patients who received a valve on the basis of measurements performed using TEE, MSCT showed that CoreValve devices exhibited low degree of oversizing $(10.1 \pm 8.6 \%)$. He also stated that choosing the valve on the basis of measurements of the annulus diameter obtained from MSCT may prompt the decision to change the previously selected valve for as many as $50 \%$ of patients. Hayashida et al. [13] studied a group of 175 patients who had both CoreValve and ES valves implanted on the basis of annulus measurements performed using MSCT and found that the annulus diameter and the median size of the implanted valves were bigger in comparison to a group of patients who had valves selected on the basis of TEE measurements. Moreover, in 34 (18.5\%) patients MSCT test led to a decision to change the valve size. For all patients, except one, the change involved implanting a bigger valve.

This study does not give clear indications as to the degree of oversizing at which the absence 
of PVL can be expected. It was however, shown that there was a statistically significant difference in valve oversizing in Group I and II. In Group II, where valve adjustment was bigger, oversizing was bigger as well. Additionally, statistically significant differences were found in the degree of valve oversizing in patients with different degrees of PVL. In general, the lower the leak, the greater the oversizing. Mylotte et al. [26] determined the minimal valve oversizing as $9.0 \%$ and $9.6 \%$ for 26 and 29 CoreValve, respectively. Buzatti et al. [27] showed that, in general, oversising of $7 \%$ reduced PVL risk, in cases of ES valve that value is $2 \%$, and in case of CoreValve - 11\%. Detaint et al. [22] concluded that for ES valve PVL does not occur when the degree of oversizing was greater than $8 \%$. Leber et al. [23] stated that PVL occurs most rarely when ES valve oversizing was $>25 \%$ of the surface area of valve annulus. Nevertheless, such oversizing was connected with higher risk of necessity of pacemaker implantation. They considered the oversizing to the degree of $15-25 \%$ over the valve surface area or $7-12 \%$ over the diameter of valve annulus to be optimal. The concerns regarding the degree of oversizing requires further studies.

Nevertheless, based on the results of this study, it was determined that the valve selection based on the diameter calculated from MSCT can offer a high level of valve tightness.

\section{Limitations of the study}

The limitation of this study is the single-center character. According to the authors, the potential impact of the learning curve has been limited by not including the first 12 patients with TAVI and also by the fact that $70 \%$ of the procedures were overseen by experienced cardiologists-proctors who had access to the results of ultrasound, angiography and MSCT examinations and the authors relied on their experience in selecting the valve size. In Group II, none of the procedures were proctored.

\section{Conclusions}

The selection of a CoreValve device based on MSCT measurement resulted in a smaller percentage of PVL, better valve adjustment and a lower incidence of composite endpoint. In almost $1 / 3$ of patients valve size changes were made. The degree of oversizing had a significant influence on paravalvular leak.

Conflict of interest: None declared

\section{References}

1. Vahanian A, Alfieri O, Andreotti F, et al. Joint Task Force on the Management of Valvular Heart Disease of the European Society of Cardiology (ESC), European Association for CardioThoracic Surgery (EACTS). Guidelines on the management of valvular heart disease (version 2012). Eur. Heart J. 2012; 33(19): 2451-2496, doi: 10.1093/eurheartj/ehs109, indexed in Pubmed: 22922415.

2. Piazza N, Kalesan B, van Mieghem N, et al. A 3-center comparison of 1-year mortality outcomes between transcatheter aortic valve implantation and surgical aortic valve replacement on the basis of propensity score matching among intermediate-risk surgical patients. JACC Cardiovasc Interv. 2013; 6(5): 443-451, doi: 10.1016/j.jcin.2013.01.136, indexed in Pubmed: 23702009.

3. Kodali SK, Williams MR, Smith CR, et al. PARTNER Trial Investigators. Two-year outcomes after transcatheter or surgical aortic-valve replacement. N. Engl. J. Med. 2012; 366(18): 1686-1695, doi: 10.1056/NEJMoa1200384, indexed in Pubmed: 22443479.

4. Athappan G, Patvardhan E, Tuzcu EM, et al. Incidence, predictors, and outcomes of aortic regurgitation after transcatheter aortic valve replacement: meta-analysis and systematic review of literature. J. Am. Coll. Cardiol. 2013; 61(15): 1585-1595, doi: 10.1016/j.jacc.2013.01.047, indexed in Pubmed: 23500308.

5. Généreux P, Head SJ, Hahn R, et al. Paravalvular leak after transcatheter aortic valve replacement: the new Achilles' heel? A comprehensive review of the literature. J. Am. Coll. Cardiol. 2013; 61(11): 1125-1136, doi: 10.1016/j.jacc.2012.08.1039, indexed in Pubmed: 23375925.

6. Leon MB, Smith CR, Mack M, et al. PARTNER Trial Investigators. Transcatheter aortic-valve implantation for aortic stenosis in patients who cannot undergo surgery. N. Engl. J. Med. 2010; 363(17): 1597-1607, doi: 10.1056/NEJMoa1008232, indexed in Pubmed: 20961243.

7. Smith CR, Leon MB, Mack MJ, et al. PARTNER Trial Investigators. Transcatheter versus surgical aortic-valve replacement in high-risk patients. N. Engl. J. Med. 2011; 364(23): 2187-2198, doi: 10.1056/NEJMoa1103510, indexed in Pubmed: 21639811.

8. Sherif MA, Abdel-Wahab M, Stöcker B, et al. Anatomic and procedural predictors of paravalvular aortic regurgitation after implantation of the Medtronic CoreValve bioprosthesis. J. Am. Coll. Cardiol. 2010; 56(20): 1623-1629, doi: 10.1016/j. jacc.2010.06.035, indexed in Pubmed: 21050971.

9. Abdel-Wahab M, Zahn R, Horack M, et al. German transcatheter aortic valve interventions registry investigators. Aortic regurgitation after transcatheter aortic valve implantation: incidence and early outcome. Results from the German transcatheter aortic valve interventions registry. Heart. 2011; 97(11): 899-906, doi: 10.1136/hrt.2010.217158, indexed in Pubmed: 21398694.

10. John D, Buellesfeld L, Yuecel S, et al. Correlation of Device landing zone calcification and acute procedural success in patients undergoing transcatheter aortic valve implantations with the self-expanding CoreValve prosthesis. JACC Cardiovasc Interv. 2010; 3(2): 233-243, doi: 10.1016/j.jcin.2009.11.015, indexed in Pubmed: 20170883.

11. Takagi K, Latib A, Al-Lamee R, et al. Predictors of moderate-tosevere paravalvular aortic regurgitation immediately after CoreValve implantation and the impact of postdilatation. Catheter Cardiovasc Interv. 2011; 78(3): 432-443, doi: 10.1002/ccd.23003, indexed in Pubmed: 21793168. 
12. Schewel D, Frerker C, Schewel J, et al. Clinical impact of paravalvular leaks on biomarkers and survival after transcatheter aortic valve implantation. Catheter Cardiovasc Interv. 2015; 85(3): 502-514, doi: 10.1002/ccd.25295, indexed in Pubmed: 24259366.

13. Hayashida K, Lefèvre T, Chevalier B, et al. Impact of post-procedural aortic regurgitation on mortality after transcatheter aortic valve implantation. JACC Cardiovasc Interv. 2012; 5(12): 1247-1256, doi: 10.1016/j.jcin.2012.09.003, indexed in Pubmed: 23257373.

14. Gurvitch R, Webb JG, Yuan R, et al. Aortic annulus diameter determination by multidetector computed tomography: reproducibility, applicability, and implications for transcatheter aortic valve implantation. JACC Cardiovasc Interv. 2011; 4(11): 1235-1245, doi: 10.1016/j.jcin.2011.07.014, indexed in Pubmed: 22115665.

15. Willson AB, Webb JG, Labounty TM, et al. 3-dimensional aortic annular assessment by multidetector computed tomography predicts moderate or severe paravalvular regurgitation after transcatheter aortic valve replacement: a multicenter retrospective analysis. J. Am. Coll. Cardiol. 2012; 59(14): 1287-1294, doi: 10.1016/j.jacc.2011.12.015, indexed in Pubmed: 22365423.

16. Binder RK, Webb JG, Willson AB, et al. The impact of integration of a multidetector computed tomography annulus area sizing algorithm on outcomes of transcatheter aortic valve replacement: a prospective, multicenter, controlled trial. J. Am. Coll. Cardiol. 2013; 62(5): 431-438, doi: 10.1016/j.jacc.2013.04.036, indexed in Pubmed: 23684679.

17. Sellers R, Levy M, Amplatz K, et al. Left retrograde cardioangiography in acquired cardiac disease. The American Journal of Cardiology. 1964; 14(4): 437-447, doi: 10.1016/00029149(64)90027-x.

18. Zamorano JL, Badano LP, Bruce C, et al. EAE/ASE recommendations for the use of echocardiography in new transcatheter interventions for valvular heart disease. Eur. Heart J. 2011; 32(17): 2189-2214, doi: 10.1093/eurheartj/ehr259, indexed in Pubmed: 21885465.

19. Lang RM, Bierig M, Devereux RB, et al. Chamber Quantification Writing Group, American Society of Echocardiography's Guidelines and Standards Committee, European Association of Echocardiography. Recommendations for chamber quantification: a report from the American Society of Echocardiography's Guidelines and Standards Committee and the Chamber Quantification Writing Group, developed in conjunction with the European Association of Echocardiography, a branch of the European Society of Cardiology. J Am Soc Echocardiogr. 2005; 18(12): 1440-1463, doi: 10.1016/j.echo.2005.10.005, indexed in Pubmed: 16376782.

20. Koos R, Mahnken AH, Dohmen G, et al. Association of aortic valve calcification severity with the degree of aortic regurgitation after transcatheter aortic valve implantation. Int. J. Cardiol. 2011; 150(2): 142-145, doi: 10.1016/j.ijcard.2010.03.004, indexed in Pubmed: 20350770.

21. Leber AW, Kasel M, Ischinger T, et al. Aortic valve calcium score as a predictor for outcome after TAVI using the CoreValve revalving system. Int. J. Cardiol. 2013; 166(3): 652-657, doi: 10.1016/j.ijcard.2011.11.091, indexed in Pubmed: 22197118.

22. Détaint D, Lepage L, Himbert D, et al. Determinants of significant paravalvular regurgitation after transcatheter aortic valve: implantation impact of device and annulus discongruence. JACC Cardiovasc Interv. 2009; 2(9): 821-827, doi: 10.1016/j. jcin.2009.07.003, indexed in Pubmed: 19778769.

23. Leber AW, Eichinger W, Rieber J, et al. MSCT guided sizing of the Edwards Sapien XT TAVI device: impact of different degrees of oversizing on clinical outcome. Int. J. Cardiol. 2013; 168(3): 2658-2664, doi: 10.1016/j.ijcard.2013.03.030, indexed in Pubmed: 23587400.

24. von Aspern K, Foldyna B, Etz CD, et al. Effective diameter of the aortic annulus prior to transcatheter aortic valve implantation: influence of area-based versus perimeter-based calculation. Int J Cardiovasc Imaging. 2015; 31(1): 163-169, doi: 10.1007/s10554014-0527-4, indexed in Pubmed: 25165022.

25. Schultz CJ, Weustink A, Piazza N, et al. Geometry and degree of apposition of the CoreValve ReValving system with multislice computed tomography after implantation in patients with aortic stenosis. J. Am. Coll. Cardiol. 2009; 54(10): 911-918, doi: 10.1016/j.jacc.2009.04.075, indexed in Pubmed: 19712801.

26. Mylotte D, Dorfmeister M, Elhmidi Y, et al. TCT-745 CoreValve Oversizing Using Multislice Computed Tomography and Clinical Outcomes: A Comparison with Transesophageal Echocardiography. Journal of the American College of Cardiology. 2013; 62(18): B227, doi: 10.1016/j.jacc.2013.08.1497.

27. Buzzatti N, Maisano F, Latib A, et al. Computed tomographybased evaluation of aortic annulus, prosthesis size and impact on early residual aortic regurgitation after transcatheter aortic valve implantation. Eur J Cardiothorac Surg. 2013; 43(1): 43-50; discussion 50, doi: 10.1093/ejcts/ezs155, indexed in Pubmed: 22551969. 